\section{Response to: 'Efficacy and improved tolerability of combination therapy with interleukin-1 blockade and MAPK pathway inhibitors for the treatment of Erdheim-Chester disease' by Campochiaro et al}

We read with interest the letter by Campochiaro et al ${ }^{1}$ that reported the efficacy of a combination regimen including anakinra and targeted therapy for the treatment of Erdheim-Chester disease (ECD), a rare multisystem inflammatory histiocytosis. Based on the involvement of inflammatory cytokines, including tumour necrosis factor-alpha and interleukin-1, in the pathophysiology and the clinical manifestations of ECD, previous reports demonstrated a variable efficacy with a good tolerance of daily subcutaneous anakinra and infliximab for the treatment of ECD. ${ }^{23}$ Among 262 patients with ECD who have been seen at our institution until 2019, 31 (12\%) (including 12 described in a previous analysis $\left.{ }^{2}\right)$ were treated with $100 \mathrm{mg}(\mathrm{n}=27)$ or $200 \mathrm{mg}(\mathrm{n}=4)$ daily of anakinra, with a mean duration of 29 months (range, 1-132). Patients were followed on a regular basis and evaluated as previously described. ${ }^{4}$ The clinical and molecular details are provided in table 1 . Patients treated with anakinra had more osseous and less central nervous system (CNS) involvements. The patients were treated with anakinra alone or in combination with steroids in two patients, and interferon-alpha in one patient. In the latter, failure of treatment led to the prescription of vemurafenib, a BRAF inhibitor, while anakinra was progressively stopped. She thus received a combination therapy (vemurafenib and anakinra) during 1 month without adverse event. Among the 31 patients, anakinra was well tolerated (apart from mild pain at the injection site) in 27 patients. Four (13\%) presented severe side effects (sepsis in two, oedema in one and heart failure in one). The global efficacy could be assessed in 24 patients (77\%). Ten

Table 1 Clinical and molecular characteristics of patients with Erdheim-Chester disease (ECD)

\begin{tabular}{|c|c|c|c|}
\hline & All $(n=262)$ & $\begin{array}{l}\text { Anakinra } \\
(n=31)\end{array}$ & $\begin{array}{l}\text { Targeted } \\
\text { therapies } \\
(\mathrm{n}=117)\end{array}$ \\
\hline $\begin{array}{l}\text { Age at diagnosis, years } \\
\text { (mean, SD) }\end{array}$ & $57.7(14.5)$ & $55.2(16.4)$ & $57.2(13.8)$ \\
\hline Sex (male/female) & $179 / 83(2.2)$ & $18 / 13(1.4)$ & $74 / 43(1.7)$ \\
\hline$B R A F^{\mathrm{V} 600 \mathrm{E}}$ & $148 / 228(65 \%)$ & $16 / 24(67 \%)$ & $95 / 116(82 \%)$ \\
\hline \multicolumn{4}{|l|}{ ECD involvements } \\
\hline Long bones & $206(79 \%)$ & $27(87 \%)$ & $101(86 \%)$ \\
\hline Cardiac & $133(51 \%)$ & $15(48 \%)$ & $83(71 \%)$ \\
\hline Aorta & $159(61 \%)$ & $17(55 \%)$ & $85(73 \%)$ \\
\hline CNS & $96(37 \%)$ & $8(26 \%)$ & $56(48 \%)$ \\
\hline Xanthelasma & $58(22 \%)$ & $7(23 \%)$ & $30(26 \%)$ \\
\hline Retroperitoneal fibrosis & $164(63 \%)$ & $20(65 \%)$ & $84(72 \%)$ \\
\hline Lung & $93(35 \%)$ & $9(29 \%)$ & $46(39 \%)$ \\
\hline \multicolumn{4}{|l|}{ Treatments } \\
\hline IFN-alpha & $164(63 \%)$ & $25(81 \%)$ & $63(54 \%)$ \\
\hline Anakinra & $31(12 \%)$ & $31(100 \%)$ & $11(9 \%)$ \\
\hline Targeted therapies & $117(45 \%)$ & $11(35 \%)$ & $117(100 \%)$ \\
\hline Deaths & $85(32 \%)$ & $10(12 \%)$ & $25(21 \%)$ \\
\hline $\begin{array}{l}\text { Median survival from } \\
\text { diagnosis (months) }\end{array}$ & 139 & 199 & Undefined \\
\hline
\end{tabular}

CNS, central nervous system; IFN, interferon.
(42\%) underwent an improvement of clinical and/or metabolic disease, in particular at osseous sites, with an objective partial or complete response on bones hypermetabolisms in 8 (33\%). Six patients (25\%) were stable, whereas 8 (33\%) experienced a progression of their disease while under anakinra, including a progression or occurrence of CNS $(n=5)$, cardiac $(n=3$, with tamponade in 2$)$, lung $(n=3)$ and/or skin $(n=2)$ disease. After receiving anakinra, 11 patients (35\%) were treated with targeted therapy, including a BRAF inhibitor in eight cases and/ or a MEK inhibitor in seven cases. The targeted therapy was efficacious in all cases. Among the whole cohort of 262 patients, 117 patients (45\%) were treated with targeted therapies (vemurafenib or dabrafenib for BRAF inhibition, and trametinib or cobimetinib for MEK inhibition). Although these patients had more cardiac and CNS involvements, they had a better survival than the patients who were not treated with targeted therapies (HR $0.6350,95 \%$ CI 0.4170 to $0.9945, \mathrm{p}=0.04$ ). Apart from a single patient who had a short combination with anakinra for 1 month described above, we did not combine targeted therapy and anakinra in our cohort.

Campochiaro et al hypothesised that anakinra could dampen the toxicity related to targeted therapies. Most adverse events occurring during BRAF inhibitor treatment, with or without MEK inhibitors, are mild or moderate and can be managed with careful monitoring, dose reduction and supportive care. ${ }^{5}$ The most severe adverse events ( $\geq$ grade 3 ) usually occur during the first cycle of treatment. Even if the addition of a MEK inhibitor to BRAF inhibition improves outcomes and decreases the incidence of squamous skin cancers and other skin-related toxicities, it is well known that cardiovascular adverse events are more frequent with combined MEK and BRAF inhibitors than with BRAF inhibitors alone (particularly the decrease in left ventricular ejection fraction). ${ }^{6}$ The mechanisms of such cardiac toxicities are not fully understood but MAPK has been shown protective for the heart. Although Campochiaro et al reported the improvement of vemurafenib-induced myocarditis with anakinra, as it was previously reported in non-toxic myocarditis, ${ }^{7}$ we believe that further evaluation is needed to use this treatment for toxic myocarditis. Recent BRAF and MEK inhibitors, encorafenib and binimetinib, have less frequent adverse events, which are usually manageable, reversible and infrequently associated with discontinuation. Notably, fever was less frequently seen than with classical BRAF and MEK inhibitors in a trial of 570 patients. ${ }^{8}$ Such drugs could represent interesting drugs for ECD treatment and should be further evaluated.

Previous studies reported the efficacy of therapies targeting the MAPK pathway for treating ECD, with patients treated after anakinra failure. ${ }^{9}$ Even if anakinra proved effective in patients with mild disease, a subset of patients experienced a progression of their disease with life-threatening manifestations including tamponade in two and CNS in five. We therefore believe that anakinra should be restricted to 'mild forms' of ECD, in particular patients with bone disease, because of its acceptable profile of tolerance and efficacy for this specific involvement. Patients should be regularly assessed to detect the occurrence of organ dysfunction (cardiac or CNS) that can occur despite anakinra, and then switched to targeted therapies if appropriate. For patients who receive targeted therapies, the security profile requires regular monitoring of heart, skin and retina. The benefit of adding anakinra for improving these toxicities remains to be established.

Fleur Cohen-Aubart $\odot,{ }^{1}$ Neila Benameur, ${ }^{2}$ Zahir Amoura, ${ }^{1}$ Julien Haroche ${ }^{1}$ 
'Sorbonne Université, Assistance Publique Hôpitaux de Paris, Service de Médecine Interne 2, Hôpital de la Pitié-Salpêtrière, Centre National de Référence Lupus Systémique et Histiocytoses, Paris, France

${ }^{2}$ Assistance Publique Hôpitaux de Paris, Département de Pharmacie, Hôpital de la pitié-Salpêtrière, Paris, France

Correspondence to Dr Fleur Cohen-Aubart, AP-HP, Hôpital de la Pitié-Salpêtrière, Service de Médecine Interne 2, Sorbonne Université Faculté de Médecine, Paris, Île-de-France 75013, France; fleur.cohen@psl.aphp.fr

Handling editor Josef S Smolen

Contributors Conception and design: FC-A and JH. Generation of clinical data: FC-A, NB and JH. Statistical analysis: FC-A. Analysis and interpretation of the data: FC-A, NB, ZA and JH. Drafting the manuscript: FC-A and JH. All authors critically reviewed and approved the final version of the manuscript.

Competing interests FC-A and JH are investigators (FC-A being the PI) of an academic study on the efficacy of cobimetinib for treating histiocytoses. They did not receive any personal fee for this. No other relevant competing interest was declared.

Patient consent for publication Not required.

Provenance and peer review Commissioned; internally peer reviewed.

(c) Author(s) (or their employer(s)) 2020. No commercial re-use. See rights and permissions. Published by BMJ.

\section{A) Check for updates}

To cite Cohen-Aubart F, Benameur N, Amoura Z, et al. Ann Rheum Dis Epub ahead of print: [please include Day Month Year]. doi:10.1136/annrheumdis-2019-216755

Received 31 December 2019

Revised 2 January 2020

Accepted 7 January 2020
- http://dx.doi.org/10.1136/annrheumdis-2019-216610

Ann Rheum Dis 2020;0:1-2. doi:10.1136/annrheumdis-2019-216755

\section{ORCID iD}

Fleur Cohen-Aubart http://orcid.org/0000-0002-7610-4995

\section{REFERENCES}

1 Campochiaro C, Cavalli G, Farina N, et al. Efficacy and improved tolerability of combination therapy with interleukin-1 blockade and MAPK pathway inhibitors for the treatment of Erdheim-Chester disease. Ann Rheum Dis 2019. doi:10.1136/ annrheumdis-2019-216610. [Epub ahead of print: 9 Dec 2019].

2 Cohen-Aubart F, Maksud P, Saadoun D, et al. Variability in the efficacy of the IL1 receptor antagonist anakinra for treating Erdheim-Chester disease. Blood 2016;127:1509-12.

3 Cohen-Aubart F, Maksud P, Emile J-F, et al. Efficacy of infliximab in the treatment of Erdheim-Chester disease. Ann Rheum Dis 2018;77:1387-90.

4 Cohen-Aubart F, Emile J-F, Carrat F, et al. Phenotypes and survival in Erdheim-Chester disease: results from a 165-patient cohort. Am J Hematol 2018;93:E114-7.

5 Dréno B, Ribas A, Larkin J, et al. Incidence, course, and management of toxicities associated with cobimetinib in combination with vemurafenib in the coBRIM study. Ann Oncol 2017:28:1137-44.

6 Mincu RI, Mahabadi AA, Michel L, et al. Cardiovascular adverse events associated with BRAF and MEK inhibitors: a systematic review and meta-analysis. JAMA Netw Open 2019;2:e198890.

7 Cavalli G, Pappalardo F, Mangieri A, et al. Treating life-threatening myocarditis by blocking interleukin-1. Crit Care Med 2016;44:e751-4.

8 Gogas HJ, Flaherty KT, Dummer R, et al. Adverse events associated with encorafenib plus binimetinib in the COLUMBUS study: incidence, course and management. Eur J Cancer 2019;119:97-106.

9 Schirmer JH, Thorns C, Moosig F, et al. Treatment failure by canakinumab in a patient with progressive multisystemic Erdheim-Chester disease refractory to anakinra: successful use of vemurafenib: fig. 1. Rheumatology 2015;54:1932-4. 